\title{
Autophagy flux inhibition augments gastric cancer resistance to the anti-human epidermal growth factor receptor 2 antibody trastuzumab
}

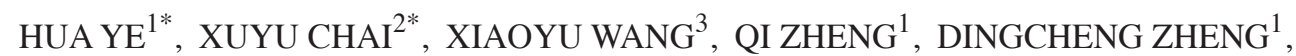 \\ FENG WU $^{1}$, CHENG ZHENG ${ }^{1}$ and PING CHEN ${ }^{1}$ \\ ${ }^{1}$ Department of Gastrointestinal and Hernia, Ningbo No. 2 Hospital, Ningbo, Zhejiang 315010; \\ ${ }^{2}$ National Pharmaceutical Engineering Research Center, China State Institute of Pharmaceutical Industry; ${ }^{3}$ Laboratory of \\ Immunology and Virology, Experiment Center for Science and Technology, \\ Shanghai University of Traditional Chinese Medicine, Shanghai 201203, P.R. China
}

Received November 6, 2016; Accepted November 2, 2017

DOI: $10.3892 / \mathrm{ol} .2018 .7891$

\begin{abstract}
The autophagy involved in the occurrence, development and prognosis of human epidermal growth factor receptor 2 (HER2) gene-amplified cancer also controls the resistance of this type of cancer to the monoclonal antibody, trastuzumab (Tzb). In the present study, Tzb resistance was established in HER2-positive NCI-N87 cell lines (Tzb-refractory cells). The cell viability, clonogenic assay, ratios of light chain $3 \mathrm{II} / \mathrm{I}$, sequestosome 1 expression, and the phosphorylation of protein kinase $\mathrm{B}(\mathrm{Akt})$ and mechanistic target of rapamycin (mTOR) were investigated in the parental and Tzb-refractory cells. The viability of parental NCI-N87 and Tzb-refractory cells with an autophagy inhibitor or inducer was also examined. The results of the present study indicated that autophagic flux may have an important function in the resistance of HER2-positive human gastric cancer NCI-N87 cells to Tzb. Tzb resistance in NCI-N87 cells prevents cell apoptosis via autophagic flux inhibition. Tzb may activate the Akt/mTOR pathway to inhibit autophagic flux in gastric cancer cell lines. Everolimus, an mTOR inhibitor, may inhibit cell viability, indicating that the mTOR pathway may serve a function in HER2-positive gastric cancer and that the resistance of HER2-positive gastric cancer to Tzb may, at least partially, be due to activation of the mTOR pathway.
\end{abstract}

Correspondence to: Professor Ping Chen, Department of Gastrointestinal and Hernia, Ningbo No. 2 Hospital, 41 Xibei Street, Ningbo, Zhejiang 315010, P.R. China

E-mail: 108156968@qq.com

${ }^{*}$ Contributed equally

Key words: autophagy, human epidermal growth factor receptor 2, trastuzumab, mechanistic target of rapamycin

\section{Introduction}

Gastric cancer is the one of most common causes of cancer-associated mortality globally and clinical approaches for treatment of gastric cancer are limited, with the median survival time for advanced gastric cancer remaining at 8-10 months (1). Dysplasia and Barrett's esophagus have been reported to be associated with the development of esophageal adenocarcinoma, while Helicobacter pylori infection, atrophic gastritis, intestinal metaplasia and dysplasia are associated with gastric adenocarcinoma (2). In $20-30 \%$ of gastric and gastro-esophageal junction cancer cases, gastric cells overexpress human epidermal growth factor receptor 2 (HER2), which is indicative of a poor prognosis (3).

Trastuzumab (Tzb) is a humanized monoclonal antibody that targets the HER2 gene. Tzb is one of the first molecular-targeting drugs to be developed and was originally introduced for the treatment of HER2-positive advanced breast cancer (4). Tzb has also been widely used to treat HER2-positive gastric cancer (1). Tzb, induces antibody-dependent cellular cytotoxicity and confers an overall survival benefit in HER2-positive advanced gastric cancer (3). However, Tzb treatment remains under investigation in order to further elucidate its potential utilization and underlying mechanisms (5). Tzb in combination with chemotherapy may be considered as a novel standard option for patients with HER2-positive advanced gastric or gastro-esophageal junction cancer (6). However, with increased durations of Tzb treatment, the risk of developing resistance to the drug is also increased. In addition, details of the mechanisms underpinning Tzb resistance remain unclear. Therefore, it is important to explore the mechanisms underlying drug resistance in order to combat this problem.

Autophagy is the cellular degradation process in which cellular proteins and organelles are engulfed by double-membrane autophagosomes and are degraded in lysosomes (7). Perturbations in autophagy have been observed in gastric cancer $(8,9)$. In cancer cells, autophagy has both pro-survival and pro-death functions and, thus, the action of autophagy in cancer cells remains controversial. Autophagy 
may act as a survival mechanism that provides energy and protects cancer cells from the cell death induced by multiple antitumor treatments; however, autophagy is also a cell death mechanism in response to anticancer therapies (10). Furthermore, autophagy modulates the development of gastric cancer by affecting a range of pathological events, including tumor angiogenesis and changes to the tumor microenvironment (11).

$\mathrm{Wu}$ et al (10) revealed that loss of the autophagy regulator beclin 1 is significantly correlated with HER2 amplification in patients with breast cancer. Notably, HER2 signaling and responsiveness to Tzb appear to dynamically interact with the tumor-suppressive and tumorigenic functions of autophagy (12). Previously, autophagy has been reported to protect against Tzb-induced cytotoxicity in HER2-overexpressing breast tumor spheroids (13). A study has revealed that the autophagy inhibitor, chloroquine, overcomes Tzb resistance in HER2-positive breast cancer SK-BR3 cells and have confirmed that HER2-overexpressing breast cancer cells may require autophagy in order to maintain the Tzb-resistant phenotype (14). However, these studies are focused on breast cancer, with only limited data regarding the association between autophagy and HER2 expression in gastric adenocarcinoma being reported. The present study investigated the function of autophagic flux in a Tzb-resistant gastric cancer cell line in order to study its mechanism of action.

\section{Materials and methods}

Materials. Tzb was provided by Ningbo No. 2 Hospital (Zhejiang, China), solubilized in water (stock solution at $21 \mathrm{mg} / \mathrm{ml}$ ), stored at $4^{\circ} \mathrm{C}$ and used within 1 month. Dimethylsulfoxide (DMSO), 3-methyladenine (3MA), MTT, crystal violet, hydroxychloroquine (HCQ) and bafilomycin A1 (BafA1) were purchased from Sigma-Aldrich; Merck KGaA (Darmstadt, Germany). Everolimus was provided by the China State Institute of Pharmaceutical Industry (Shanghai, China). RPMI-1640 medium, $10 \mathrm{U} / \mathrm{ml}$ penicillin-streptomycin $(\mathrm{P} / \mathrm{S})$, $0.25 \%$ trypsin, fetal bovine serum (FBS) and bovine serum albumin (BSA) were purchased from Invitrogen (Thermo Fisher Scientific, Inc., Waltham, MA, USA). Cell Counting Kit-8 (CCK-8) was purchased from Dojindo Molecular Technologies, Inc. (Kumamoto, Japan). Cell lysis buffer, polyvinylidene difluoride (PVDF) membranes, and Tween-20 were purchased from Weiao Inc. (Shanghai, China). Glutaraldehyde, Epon 812, DDSA, NMA and DMP-30 were purchased from Sinopharm Inc. (Beijing, China).

Cell culture. Human gastric cancer NCI-N87 and SGC 7901 cell lines, and the human breast cancer SK-BR3 cell line, which was used as the positive control, were purchased from the Shanghai Institute of Biochemistry and Cell Biology (Shanghai, China). The cells were cultured in full medium (P/S and RPMI-1640 medium, supplemented with 10\% FBS) at $37^{\circ} \mathrm{C}$ in a humidified atmosphere with $5 \% \mathrm{CO}_{2}$.

Establishment of Tzb-acquired auto-resistance in HER2-positive NCI-N87 cells. Establishment of NCI-N87/Tzb-resistant pools was performed as previously described (15), with
Tzb-naive NCI-N87 cells being treated with increasing concentrations of Tzb for 10 months. The NCI-N87 cells were initially treated with $50 \mu \mathrm{g} / \mathrm{ml}$ Tzb for 2 months (twice weekly) followed by $100 \mu \mathrm{g} / \mathrm{ml}$ Tzb for 3 months (twice weekly). Two pools (Tzb POOL1 and Tzb POOL2), which resisted continuous growth in $250 \mu \mathrm{g} / \mathrm{ml}$ Tzb for 5 months (twice weekly), were selected for further study. The resistant cells were maintained in RPMI-1640 medium without Tzb for $\geq 2$ days prior to further experiments.

MTT assay. The NCI-N87, POOL1 or POOL2 cells were seeded at 4,000 cells/100 $\mu$ l into 96-well plates, and allowed to attach overnight. The medium was removed, and $95 \mu \mathrm{l}$ fresh full medium with $5 \mu 1$ Tzb (final concentration 1, 3.2, 10, 32, $100 \mu \mathrm{g} / \mathrm{ml})$, BafA1 $(50 \mathrm{nM}), 3 \mathrm{MA}(1 \mu \mathrm{M})$, HCQ $(50 \mu \mathrm{M})$ or everolimus $(0.1,1,10 \mu \mathrm{M})$. The control cells treated with $5 \mu \mathrm{l}$ PBS was used as vehicle. For testing the combination effect of Tzb $(10 \mu \mathrm{g} / \mathrm{ml})$ and autophagy inhibitors, the concentration of BafA1, 3MA, and HCQ is $25 \mathrm{nM}, 500$ and $25 \mu \mathrm{M}$ respectively. After $72 \mathrm{~h}$ culture $\left(37^{\circ} \mathrm{C}, 5 \% \mathrm{CO}_{2}\right), 10 \mu \mathrm{l} \mathrm{MTT}$ solution $(0.5 \mathrm{mg} / \mathrm{ml})$ was added into each well at $37^{\circ} \mathrm{C}$ for $4 \mathrm{~h}$ and $100 \mu \mathrm{l}$ DMSO was used to dissolve the purple formazan. Following agitation, the optical densities were measured at $595 \mathrm{~nm}$ using a microplate reader (BioTek PowerWave XS2; BioTek Instruments, Inc., Winooski, VT, USA).

CCK- 8 assay. The NCI-N87 or POOL2 cells were seeded at 1,000 cells $/ 100 \mu 1$ into 96 well plates, and allowed to attach overnight. The medium was removed, and $95 \mu \mathrm{l}$ fresh full medium with $5 \mu 1 \mathrm{Tzb}$ (final concentration 1, 3.2, 10, 32 or $100 \mu \mathrm{g} / \mathrm{ml})$, or everolimus $(0.1,1$ or $10 \mu \mathrm{M})$. The control cells treated with $5 \mu$ l PBS was used as vehicle. When assessing the combination effect of $\mathrm{Tzb}(10 \mu \mathrm{g} / \mathrm{ml})$ and an autophagy inhibitor, the concentration of BafA1, 3MA, and HCQ is $25 \mathrm{nM}, 500$ and $25 \mu \mathrm{M}$, respectively. After a $72 \mathrm{~h}$ culture $\left(37^{\circ} \mathrm{C}, 5 \% \mathrm{CO}_{2}\right), 10 \mu \mathrm{l} \mathrm{CCK}-8$ solution was added into each well at $37^{\circ} \mathrm{C}$ for $2 \mathrm{~h}$ and the optical densities were measured at $450 \mathrm{~nm}$ using a microplate reader (PowerWave XS2; BioTek Instruments, Inc.).

Clonogenic assay. Cells were cultured in 6-well plates at a density of 100 cells/well and were incubated $\left(37^{\circ} \mathrm{C}, 5 \% \mathrm{CO}_{2}\right)$ for $24 \mathrm{~h}$ with full medium to allow for attachment. Following incubation, cells were treated with $100 \mu \mathrm{g} / \mathrm{ml} \mathrm{Tzb}$ for $72 \mathrm{~h}$. The control cells treated with $5 \mu \mathrm{l}$ PBS was used as vehicle. Allowed to grow in a Tzb-free full medium for 14 days $\left(37^{\circ} \mathrm{C}\right.$, $5 \% \mathrm{CO}_{2}$ ), fixed with cold ethanol for $15 \mathrm{~min}$ and stained with $0.1 \%$ crystal violet in PBS for $30 \mathrm{~min}$ at $25^{\circ} \mathrm{C}$. Images from light microscopy (magnification, $x 4$; Olympus IX73) were captured using CellSens Dimension software (version 1.15) (both from Olympus Corporation, Tokyo, Japan).

Western blot analysis. Protein lysates were obtained with cell lysis buffer for $30 \mathrm{~min}$ at $4^{\circ} \mathrm{C}$. The protein $(20 \mu \mathrm{g} /$ lane $)$ was fractionated by $10 \%$ SDS-PAGE and transferred onto PVDF membranes. After $1 \mathrm{~h}$ blocking with $5 \% \mathrm{BSA}$ at $25^{\circ} \mathrm{C}$, the membranes were incubated at $4^{\circ} \mathrm{C}$ overnight with different primary antibodies diluted in $3 \%$ BSA in 1\% Tween-20 in TBS. The membranes were probed with the following antibodies: HER2 (cat. no. 4290; Cell Signaling Technology, 
A

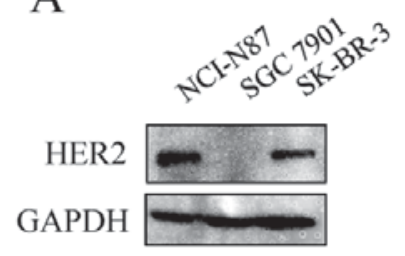

B

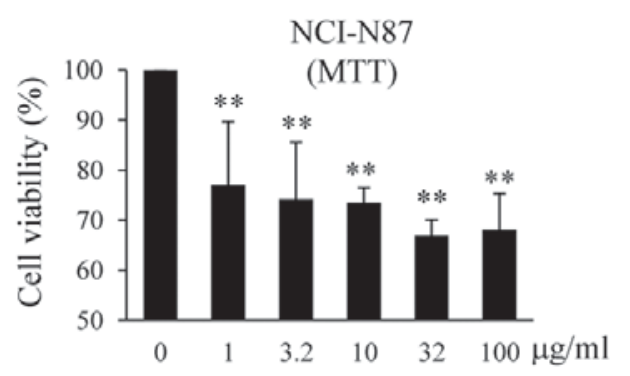

$\mathrm{D}$

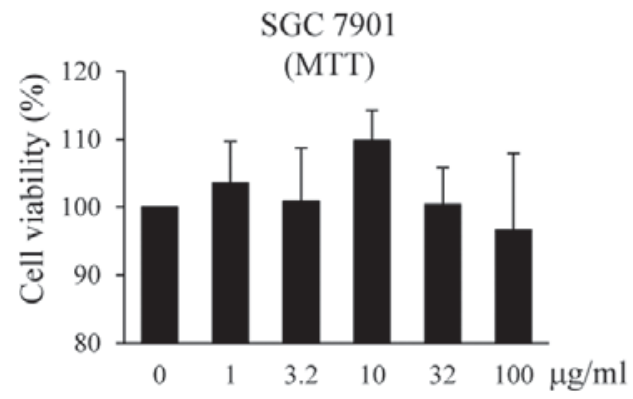

$\mathrm{C}$

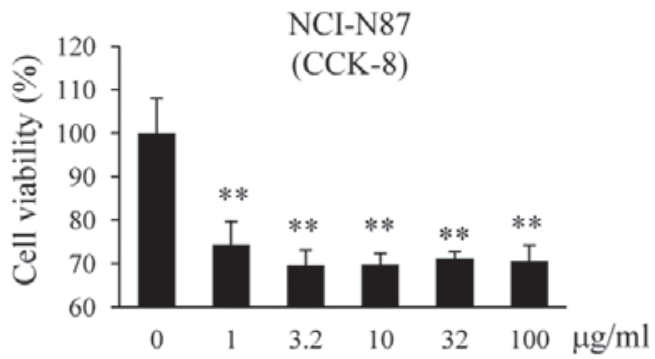

$\mathrm{E}$

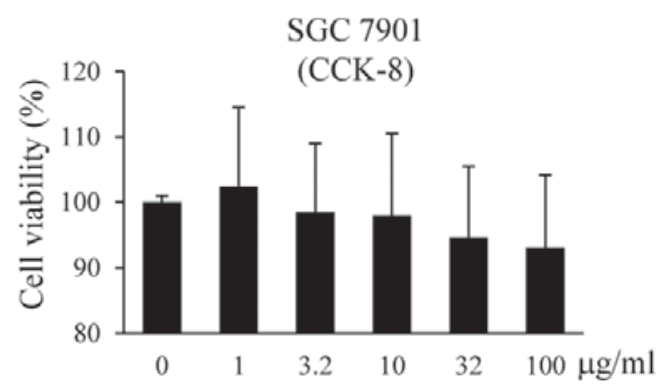

Figure 1. HER2 protein expression in NCI-N87, SGC 7901 and SKBR3 cells. (A) Cells were collected and underwent western blot analysis for HER2 and GAPDH expression. NCI-N87 cells were treated with graded concentrations of Tzb (or vehicle) and cell viability was determined using a (B) MTT assay or (C) CCK-8 assay. SGC 7901 cells were treated with graded concentrations of Tzb (or vehicle) and cell viability was determined using a (D) MTT assay or (E) CCK-8 assay. ${ }^{* *} \mathrm{P}<0.01$ vs. vehicle. HER2, human epidermal growth factor receptor 2; Tzb, trastuzumab; CCK-8, Cell Counting Kit-8.

Inc., Danvers, MA, USA; dilution 1:1,000), light chain 3B (LC3B) (cat. no. L7543; dilution 1:1,000; Sigma-Aldrich; Merck KGaA), sequestosome 1 (SQSTM1; cat. no. PM045; dilution 1:2,000; MBL International Corporation, Woburn, MA, USA), phosphorylated (p)-protein kinase B (Akt; cat. no. 2965; dilution 1:1,000), Akt (cat. no. 2966; dilution 1:1,000), p-mechanistic target of rapamycin (mTOR; cat. no. 2983; dilution 1:1,000) and mTOR (cat. no. 5536; dilution 1:1,000) (all from Cell Signaling Technology, Inc.). GAPDH (cat. no. 5014; dilution 1:2,000; Cell Signaling Technology, Inc.) was used as the reference protein. Subsequently, the membranes were incubated with horseradish peroxidase-conjugated secondary anti-rabbit IgG antibody (cat. no. 7074; dilution 1:2,000; Cell Signaling Technology, Inc.) for $1 \mathrm{~h}$ at room temperature. Protein bands were visualized using an enhanced chemiluminescence (ECL) kit using ECL blotting detection reagents (cat. no. 54-61-00; KPL, Inc., Gaithersburg, MD, USA).

Transmission electron microscopy (TEM). A total of $1 \times 10^{7}$ cells were digested with $0.25 \%$ trypsin at $37^{\circ} \mathrm{C}$ and washed twice with PBS. The cell precipitation was fixed with $2.5 \%$ glutaraldehyde in PBS for $3 \mathrm{~h}$ at $4^{\circ} \mathrm{C}$, followed by $1 \%$ $\mathrm{OsO}_{4}$ for $2 \mathrm{~h}$ at $4^{\circ} \mathrm{C}$. After washing with PBS, the samples were progressively dehydrated in a graduated series of ethanol solutions $(50,70,80,90,95$ and $100 \%)$ at $4^{\circ} \mathrm{C}$ and embedded in the mixture of Epon 812, DDSA, NMA and DMP-30 at $37^{\circ} \mathrm{C}$ for $12 \mathrm{~h}$, and at $60^{\circ} \mathrm{C}$ for $48 \mathrm{~h}$. Following dehydration, the cells were cut in to $70 \mathrm{~nm}$ sections, and stained with $3 \%$ uranyl acetate and lead citrate for $15 \mathrm{~min}$ at $25^{\circ} \mathrm{C}$, the ultrastructure of cells were analyzed with a TEM (magnification, x16,000; cat. no. JEM-1230; JEOL, Ltd., Tokyo, Japan).

Statistical analysis. All of the data are presented as the mean \pm standard deviation of three independent experiments. Statistical comparisons were performed using Student's t-test and repeated-measures one-way analysis of variance followed by Dunnett's post hoc test using SPSS software (version 19; IBM Corp., Armonk, NY, USA). P<0.05 was considered to indicate a statistically significant difference.

\section{Results}

Tzb induces gastric cancer NCI-N87 cell death. HER2 was expressed in human gastric cancer NCI-N87 cells, but not in SGC 7901 cells (Fig. 1A). Breast cancer SKBR 3 cells were used as a positive control. The viability of NCI-N87 and SGC 7901 cells treated with Tzb was examined using MTT and CCK-8 assays. Tzb (1-100 $\mu \mathrm{g} / \mathrm{ml})$ was revealed to significantly 
A

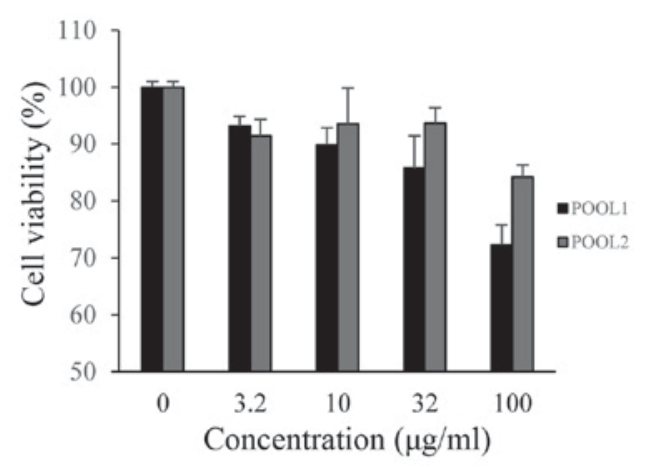

$\mathrm{C}$

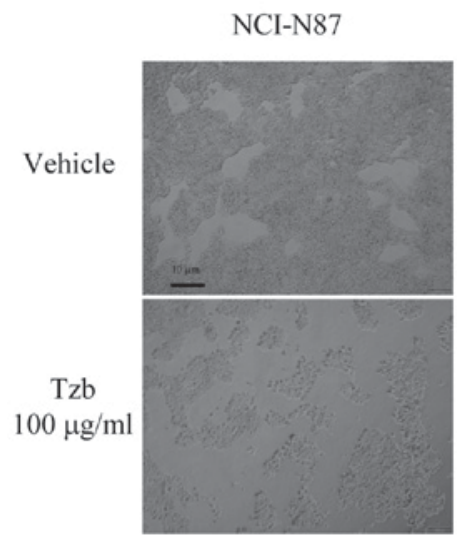

B

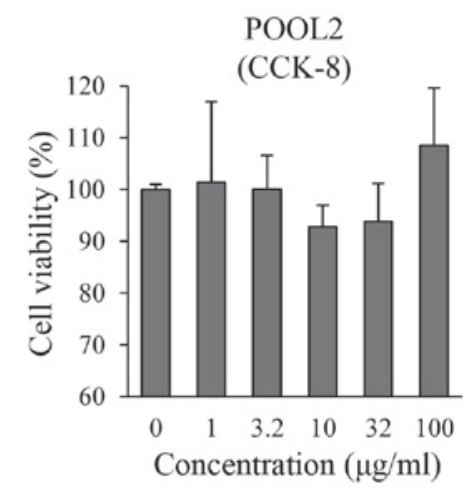

$\mathrm{D}$ 
A

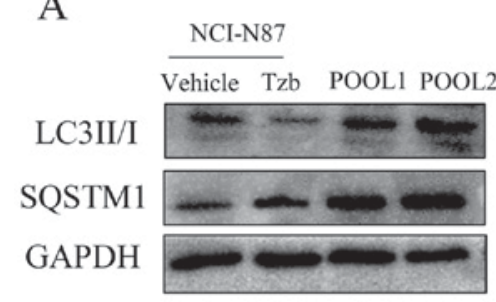

$\mathrm{C}$

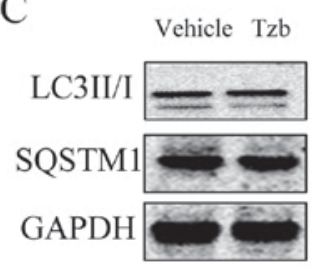

$\mathrm{B}$

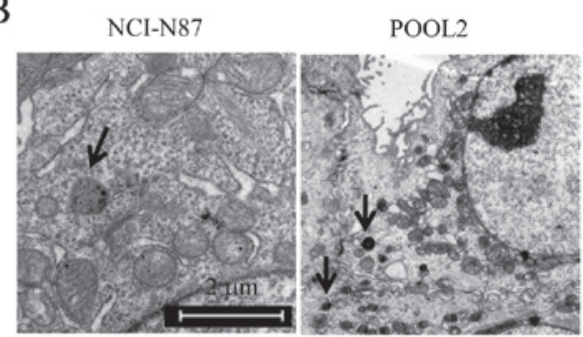

Figure 3. Dynamics of autophagy in NCI-N87 Tzb-refractory cells. (A) Autophagosome formation in whole cell lysates of Tzb-naive NCI-N87 parental cells and Tzb-refractory POOL cells was detected by western blot analysis using LC3 and SQSTM1 antibodies. (B) Representative transmission electron microscopy images depicting ultrastructures of Tzb-naive NCI-N87 parental cells and Tzb-refractory POOL2 cells. Arrows indicate autolysosomes. (C) Autophagosome formation in whole cell lysates of POOL2 cells treated with Tzb was detected by western blot analysis using LC3 and SQSTM1 antibodies. The image demonstrates a representative western blot analysis and similar results were obtained in three independent experiments. Tzb, trastuzumab; LC3, light chain 3; SQSTM1, sequestosome 1.

A

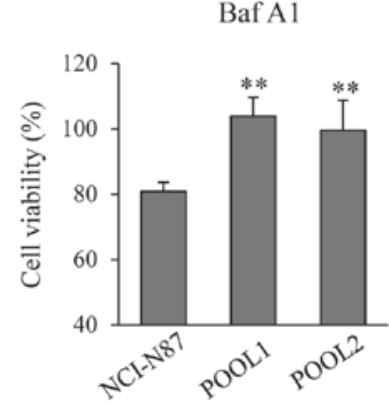

$\mathrm{D}$

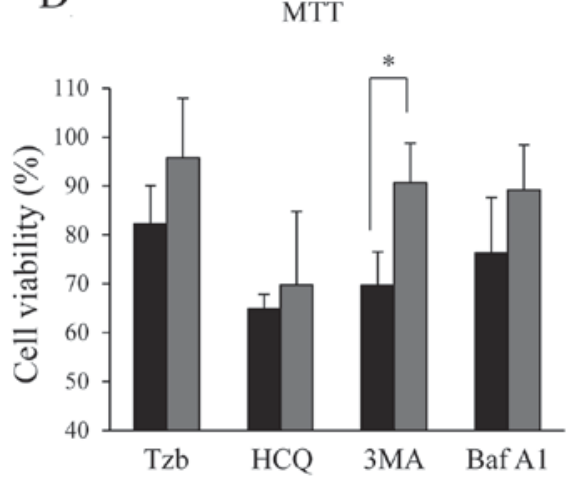

B

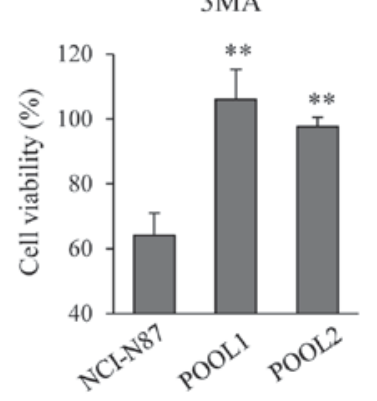

$\mathrm{C}$

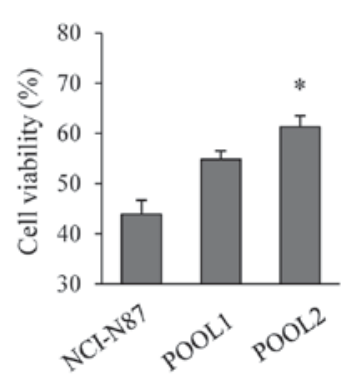

$\mathrm{E}$

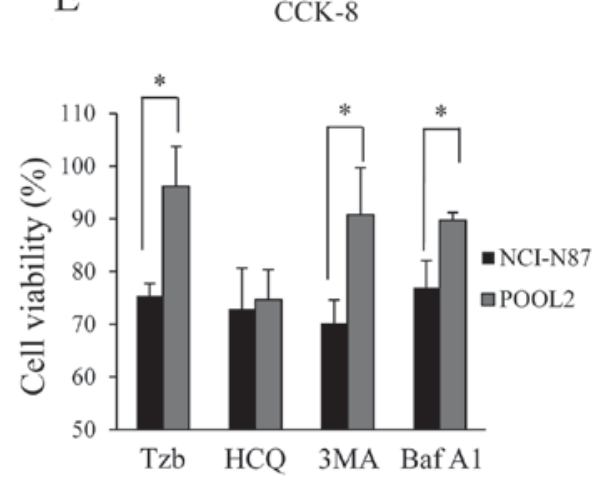

Figure 4. Cell viability of NCI-N87 and Tzb-refractory cells treated with autophagy inhibitors. The effects of treatment with (A) BafA1 (50 nM), (B) 3MA $(1 \mathrm{mM})$ and $(\mathrm{C}) \mathrm{HCQ}(50 \mu \mathrm{M})$ were determined using MTT assays. (D and E) The combination effect of Tzb and autophagy inhibitors. The viability of cells treated with Tzb $(10 \mu \mathrm{g} / \mathrm{ml})$ and autophagy inhibitors, BafA1 $(25 \mathrm{nM})$, 3MA $(500 \mu \mathrm{M})$ or HCQ $(25 \mu \mathrm{M})$, was also determined using (D) MTT assay and (E) CCK-8 assay. ${ }^{\mathrm{P}}<0.05,{ }^{\text {** }} \mathrm{P}<0.01$ vs. parental NCI-N87 cells. Tzb, trastuzumab; BafA1, bafilomycin A1; 3MA, 3-methyladenine; HCQ, hydroxychloroquine.

Tzb did not alter the autophagic flux in POOL2. These results indicated that Tzb-refractory cells exhibited less autophagic flux and improved viability compared with parental cells, suggesting that autophagy may induce the cell death of gastric cancer cells.

BafA1 is a vacuolar $\mathrm{H}^{+}$-ATPase inhibitor known to block autophagic flux to prevent fusion between autophagosomes and lysosomes, $3 \mathrm{MA}$ is an inhibitor of the formation of the pre-autophagosomal structures and HCQ is an autophagic inhibitor that prevents endosomal acidification and blocks autophagosome-lysosome fusion $(13,17,18)$. In the present study, cell viability was studied in both parental NCI-87 and Tzb-refractory cells following blocking of autophagy with BafA1 $(50 \mathrm{nM}), 3 \mathrm{MA}(1 \mathrm{mM})$ or HCQ $(50 \mu \mathrm{M})$. The three 

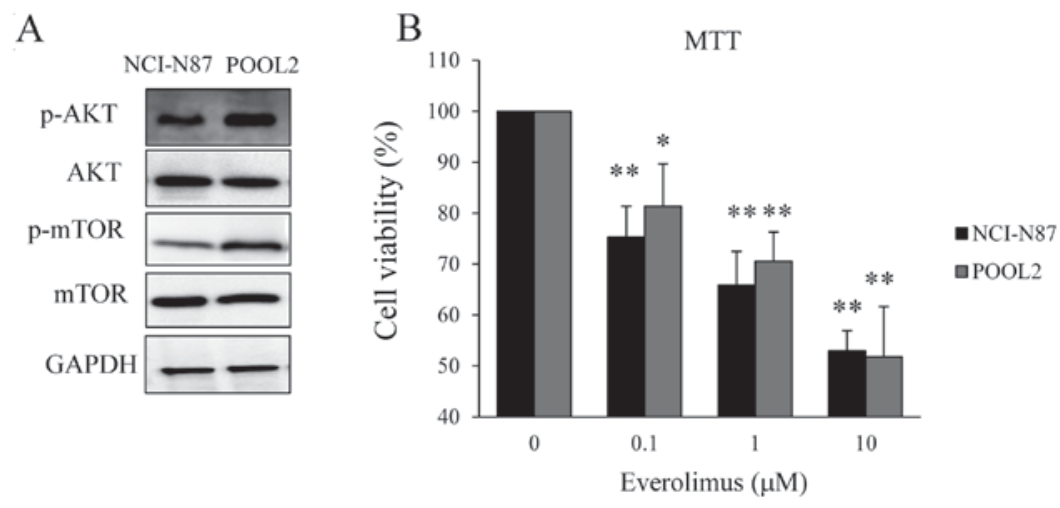

C

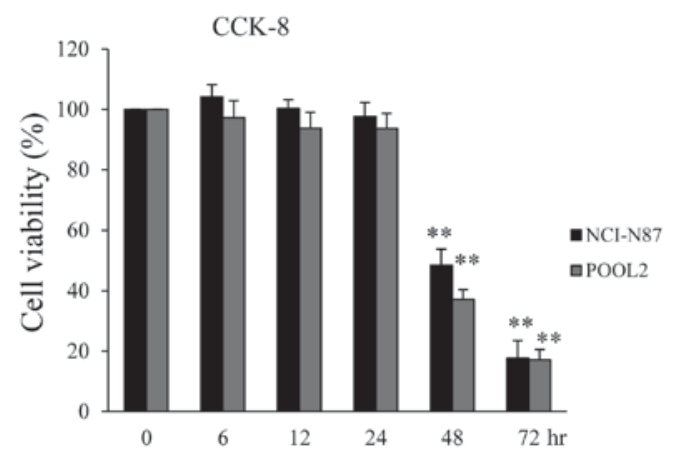

D

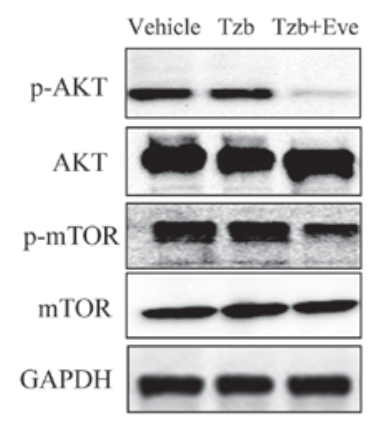

Figure 5. Tzb induces resistance via the Akt/mTOR pathway. (A) Phosphorylation of Akt and mTOR in NCI-N87 and Tzb-refractory POOL2 cells, as assessed using western blotting. (B) The viability of NCI-N87 and Tzb-refractory cells following treatment with graded concentrations of everolimus (72 h) was determined using MTT assay. (C) The viability of cells treated with $10 \mu \mathrm{M}$ everolimus for various durations was determined using CCK-8 assay. (D) The phosphorylation of Akt and mTOR following treatment with everolimus and/or Tzb in POOL2 cells. ${ }^{*} \mathrm{P}<0.05,{ }^{* * *} \mathrm{P}<0.01$ vs. vehicle. mTOR, mechanistic target of rapamycin; Tzb, trastuzumab; p, phosphorylated; CCK-8, Cell Counting Kit-8.

autophagy inhibitors, BafA1, 3MA and HCQ, were revealed to induce parental NCI-N87 cell death (Fig. 4A-C). However, no change in the viability of POOL1 or POOL2 cells treated with BafA1 or 3MA was observed, suggesting that these autophagy inhibitors did not alter the viability of Tzb-refractory cells. HCQ overcame Trz resistance, but its effect was less notable than that in the parental NCI-N87 cells. In addition, the results of MTT and CCK-8 assays revealed that the combination of Tzb (10 $\mu \mathrm{g} / \mathrm{ml})$ with an autophagy inhibitor (BafA1, 3MA or HCQ) may inhibit the growth of parental cells, but neither BafA1 nor 3MA induced Tzb-refractory cell death (Fig. 4D and E). Although HCQ may induce Tzb-refractory cell death, this effect was significantly lower than in the parental cells. These results indicated that cell Tzb resistance may be due to autophagic flux inhibition.

Tzb induces resistance via the Akt/mTOR pathway. The Akt/mTOR signaling pathway is a key negative regulator of autophagy in gastric cancer cells (8). Therefore, the present study investigated whether the Akt/mTOR pathway is involved in the Tzb resistance of HER2-positive cancer cells. As demonstrated in Fig. 5A, the phosphorylation of Akt and mTOR was increased in Tzb-resistant HER2 positive cancer cells compared with the parental cells, suggesting that Tzb resistance is partially due to alterations in the Akt/mTOR pathway. Notably, everolimus, a derivative of rapamycin that is functionally similar to rapamycin and is an allosteric inhibitor of mTOR, is also able to induce autophagy $(19,20)$, and significantly induced cell death in parental and Tzb-refractory gastric cancer cells in a dose- and time-dependent manner (Fig. 5B and C). As demonstrated in Fig. 5D, Tzb had little effect on the phosphorylation of Akt and mTOR in POOL2, however, combining everolimus with Tzb reduced the activation of Akt and mTOR. Taken together, these results suggest that Tzb-induction in cells may alter the balance of cellular autophagic flux, via autophagic flux inhibition or the use of an autophagy inducer, such as everolimus.

\section{Discussion}

Although a number of studies have reported that autophagy induction is associated with the viability of HER2-amplified human breast cancer cells in response to the anti-HER2 monoclonal antibody Tzb, few studies have investigated the association between autophagy and HER2 in gastric cancer. To the best of our knowledge, the present study is the first to investigate the function of autophagy in HER2-refractory gastric cancer.

The present study revealed that Tzb reduced the expression of LC3 I and increased the expression of SQSTM1 in NCI-N87 cells, suggesting that Tzb may inhibit autophagic flux. Tzb-refractory cells exhibited less autophagic flux and increased viability compared with the parental cancer cells, suggesting that autophagy may induce cell death in gastric cancer cells. The involvement of autophagy in tumorigenesis and cancer therapy is complex. In the present study, the 
autophagy inhibitors, BafA1 and 3MA, induced NCI-N87 cell death, but did not induce the death of Tzb-refractory cells. -HCQ was able to overcome Tzb resistance, but had a weaker effect than that observed in the parental NCI-N87 cells. These results suggest that, in Tzb-refractory NCI-N87 cells, cell apoptosis is prevented via autophagy inhibition and not autophagy activation. Certain studies have also reported that the autophagy inhibitor, chloroquine, is an effective treatment for Tzb-refractory HER2 gene-amplified metastatic breast cancer (14), which is in line with the results of the present study in NCI-N87 cells. As HCQ is not only an autophagy inhibitor, having other functions that include Toll-like receptor regulation (21) and AMP-activated protein kinase induction (22), it may induce cancer cell death via various pathways not associated with autophagic flux.

However, certain aspects of the results obtained in NCI-N87 cells in the present study are not consistent with the previous results associated with breast cancer $(13,15)$. One reason for this is that the cell lines used were different. It has been reported that HER2 protein overexpression and gene amplification are more heterogeneous in gastric cancer than in breast cancer (5). In gastric and gastroesophageal cancer, HER2 overexpression varies and prognostic relevance is inconsistent (5), which may explain, to a certain extent, the inconsistency in the mechanisms of autophagy in different Tzb-refractory cells. Additionally, Tzb resistance time and the method used to achieve Tzb resistance differ to those used previously.

The Akt/mTOR signaling pathway is an important negative regulator of autophagic flux (23-25). In the present study, the phosphorylation of Akt and mTOR in Tzb-refractory cells and parental NCI 87 cells was examined. The data demonstrated that Tzb may activate the Akt/mTOR pathway to inhibit autophagic flux.

Everolimus has been approved by the US Food and Drug Administration for the treatment of renal and breast cancer (26). The present study demonstrated that everolimus induces the cell death of parental gastric cancer cells and Tzb-refractory cells, suggesting that the mTOR inhibitor inhibits Tzb-refractory cell growth. Fuereder et al (27) reported that another mTOR inhibitor, BEZ235, inhibited the growth of NCI-N87, but not that of MK45 or MKN28 (HER2-negative gastric cancer) xenografts. Furthermore, Zhu et al (28) reported that BEZ235 exerts extensive antitumor activity in HER2-positive gastric cancer (NCI-N87 and SNU216) in vitro and in vivo. These results implied that mTOR may serve an important function in HER2-positive gastric cancer and that the resistance of HER2 to Tzb may, at least partially, be due to mTOR activation.

In conclusion, the present study suggests that autophagy may be involved in the resistance of human gastric cancer cells to Tzb. Furthermore, it was also revealed that the Akt/mTOR pathway is involved in the Tzb resistance of gastric cancer cell lines and that the autophagy inducer, everolimus, is able to inhibit cell growth in these cell lines. These results suggest that Tzb-refractory cells prevent cell apoptosis via autophagic flux inhibition, and that everolimus may overcome resistance to Tzb in HER2-postive gastric cancer. Although these effects are not fully understood and the details of such mechanisms require further investigation, autophagy inhibited by $\mathrm{Tzb}$ appears to provide cells with the capacity to evolve and develop Tzb resistance, thereby preventing gastric cancer cell death.

\section{Acknowledgements}

The present study was supported by the National Natural Science Foundation of China (grant no. 81503102), the Natural Science Foundation of Ningbo (grant no. 2015A610230), the Oncology Key Special Subject of Ningbo (grant no. 2016-B05) and the Medical Science and Technology of Clinical Research Projects in Zhejiang Province (grant no. 2015kyB345).

\section{References}

1. Wang Q, Zhang X, Shen E, Gao J, Cao F, Wang X, Li Y, Tian T, Wang J, Chen Z, et al: The anti-HER3 antibody in combination with trastuzumab exerts synergistic antitumor activity in HER2-positive gastric cancer. Cancer Lett 380: 20-30, 2016.

2. Gravalos $C$ and Jimeno A: HER 2 in gastric cancer: A new prognostic factor and a novel therapeutic target. Ann Oncol 19: 1523-1529, 2008.

3. Arienti C,Zanoni M, Pignatta S, Del Rio A, Carloni S, Tebaldi M, Tedaldi G and Tesei A: Preclinical evidence of multiple mechanisms underlying trastuzumab resistance in gastric cancer. Oncotarget 7: 18424-18439, 2016.

4. Slamon DJ, Clark GM, Wong SG, Levin WJ, Ullrich A and McGuire WL: Human breast cancer: Correlation of relapse and survival with amplification of the HER-2/neu oncogene. Science 235: 177-182, 1987.

5. Abrahao-Machado LF and Scapulatempo-Neto C: HER2 testing in gastric cancer: An update. World J Gastroenterol 22: 4619-4625, 2016.

6. Bang YJ, Van Cutsem E, Feyereislova A, Chung HC, Shen L, Sawaki A, Lordick F, Ohtsu A, Omuro Y, Satoh T, et al: Trastuzumab in combination with chemotherapy versus chemotherapy alone for treatment of HER2-positive advanced gastric or gastro-oesophageal junction cancer (ToGA): A phase 3, open-label, randomised controlled trial. Lancet 376: 687-697, 2010.

7. Zhi X and Zhong Q: Autophagy in cancer. F1000prime Rep 7: $18,2015$.

8. Zhao Z, Han F, Yang S, Wu J and Zhan W: Oxamate-mediated inhibition of lactate dehydrogenase induces protective autophagy in gastric cancer cells: Involvement of the Akt-mTOR signaling pathway. Cancer Lett 358: 17-26, 2015.

9. Pan WR, Chen YL, Hsu HC and Chen WJ: Antimicrobial peptide GW-H1-induced apoptosis of human gastric cancer AGS cell line is enhanced by suppression of autophagy. Mol Cell Biochem 400: 77-86, 2015.

10. Wu M, Lao Y, Xu N, Wang X, Tan H, Fu W, Lin Z and Xu H: Guttiferone $\mathrm{K}$ induces autophagy and sensitizes cancer cells to nutrient stress-induced cell death. Phytomedicine 22: 902-910, 2015.

11. Qian HR and Yang Y: Functional role of autophagy in gastric cancer. Oncotarget 7: 17641-17651, 2016.

12. Cufi S, Vazquez-Martin A, Oliveras-Ferraros C, CorominasFaja B, Urruticoechea A, Martin-Castillo B and Menendez JA: Autophagy-related gene 12 (ATG12) is a novel determinant of primary resistance to HER2-targeted therapies: Utility of transcriptome analysis of the autophagy interactome to guide breast cancer treatment. Oncotarget 3: 1600-1614, 2012.

13. Rodriguez CE, Reidel SI, Bal de Kier Joffé ED, Jasnis MA and Fiszman GL: Autophagy protects from trastuzumab-induced cytotoxicity in HER2 overexpressing breast tumor spheroids. PLoS One 10: e0137920, 2015.

14. Cufi S, Vazquez-Martin A, Oliveras-Ferraros C,Corominas-Faja B, Cuyàs E, López-Bonet $\mathrm{E}$, Martin-Castillo $\mathrm{B}$, Joven $\mathrm{J}$ and Menendez JA: The anti-malarial chloroquine overcomes primary resistance and restores sensitivity to trastuzumab in HER2-positive breast cancer. Sci Rep 3: 2469, 2013.

15. Vazquez-Martin A, Oliveras-Ferraros C and Menendez JA: Autophagy facilitates the development of breast cancer resistance to the anti-HER 2 monoclonal antibody trastuzumab. PLoS One 4: e6251, 2009.

16. Bartlett BJ, Isakson P, Lewerenz J, Sanchez H, Kotzebue RW, Cumming RC, Harris GL, Nezis IP, Schubert DR, Simonsen A and Finley KD: p62, $\operatorname{Ref}(2) \mathrm{P}$ and ubiquitinated proteins are conserved markers of neuronal aging, aggregate formation and progressive autophagic defects. Autophagy 7: 572-583, 2011. 
17. Pamarthy S, Jaiswal MK, Kulshreshtha A, Katara GK, Gilman-Sachs A and Beaman KD: The vacuolar ATPase a2-subunit regulates Notch signaling in triple-negative breast cancer cells. Oncotarget 6: 34206-34220, 2015

18. Manic G, Obrist F, Kroemer G, Vitale I and Galluzzi L: Chloroquine and hydroxychloroquine for cancer therapy. Mol Cell Oncol 1: e29911, 2014.

19. Lui A, New J, Ogony J, Thomas S and Lewis-Wambi J: Everolimus downregulates estrogen receptor and induces autophagy in aromatase inhibitor-resistant breast cancer cells. BMC Cancer 16: 487, 2016.

20. Lin CI, Whang EE, Donner DB, Du J, Lorch J, He F, Jiang X, Price BD, Moore FD Jr and Ruan DT: Autophagy induction with RAD001 enhances chemosensitivity and radiosensitivity through Met inhibition in papillary thyroid cancer. Mol Cancer Res 8: 1217-1226, 2010.

21. Said A, Bock S, Lajqi T, Muller G and Weindl G: Chloroquine promotes IL-17 production by $\mathrm{CD}^{+} \mathrm{T}$ cells via $\mathrm{p} 38$-dependent IL-23 release by monocyte-derived Langerhans-like cells. J Immunol 193: 6135-6143, 2014.

22. Spears LD, Tran AV, Qin CY, Hobbs SB, Burns CA, Royer NK, Zhang Z, Ralston L and Fisher JS: Chloroquine increases phosphorylation of AMPK and Akt in myotubes. Heliyon 2: e00083, 2016.

23. O'Reilly KE, Rojo F, She QB, Solit D, Mills GB, Smith D, Lane H, Hofmann F, Hicklin DJ, Ludwig DL, et al: mTOR inhibition induces upstream receptor tyrosine kinase signaling and activates Akt. Cancer Res 66: 1500-1508, 2006.
24. Yang XL, Lin FJ, Guo YJ, Shao ZM and Ou ZL: Gemcitabine resistance in breast cancer cells regulated by PI3K/AKT-mediated cellular proliferation exerts negative feedback via the MEK/MAPK and mTOR pathways. OncoTargets Ther 7: 1033-1042, 2014.

25. Zhang L, Wang H, Xu J,Zhu J and Ding K: Inhibition of cathepsin $\mathrm{S}$ induces autophagy and apoptosis in human glioblastoma cell lines through ROS-mediated PI3K/AKT/mTOR/p70S6K and JNK signaling pathways. Toxicol Lett 228: 248-259, 2014.

26. Saran U, Foti M and Dufour JF: Cellular and molecular effects of the mTOR inhibitor everolimus. Clin Sci (Lond) 129: 895-914, 2015.

27. Fuereder T, Wanek T, Pflegerl P, Jaeger-Lansky A, Hoeflmayer D, Strommer S, Kuntner C, Wrba F, Werzowa J, Hejna M, et al: Gastric cancer growth control by BEZ235 in vivo does not correlate with PI3K/mTOR target inhibition but with [18F]FLT uptake. Clin Cancer Res 17: 5322-5332, 2011.

28. Zhu Y, Tian T, Zou J, Wang Q, Li Z, Li Y, Liu X, Dong B, Li N, Gao J and Shen L: Dual PI3K/mTOR inhibitor BEZ235 exerts extensive antitumor activity in HER2-positive gastric cancer. BMC Cancer 15: 894, 2015.

This work is licensed under a Creative Commons Attribution-NonCommercial-NoDerivatives 4.0 International (CC BY-NC-ND 4.0) License. 